\title{
A CRITERION FOR HOMOGENEOUS PRINCIPAL BUNDLES
}

\author{
INDRANIL BISWAS AND GÜNTHER TRAUTMANN
}

\begin{abstract}
We consider principal bundles over $G / P$, where $P$ is a parabolic subgroup of a semisimple and simply connected linear algebraic group $G$ defined over $\mathbb{C}$. We prove that a holomorphic principal $H$-bundle $E_{H} \longrightarrow G / P$, where $H$ is a complex reductive group, is homogeneous if the adjoint vector bundle $\operatorname{ad}\left(E_{H}\right)$ is homogeneous. Fix a faithful $H$-module $V$. We also show that $E_{H}$ is homogeneous if the vector bundle $E_{H} \times{ }^{H} V$ associated to it for the $H$-module $V$ is homogeneous.
\end{abstract}

\section{IntRODUCTION}

Let $G$ be a semisimple and simply connected linear algebraic group defined over $\mathbb{C}$ and $P \subset G$ a parabolic subgroup. So the quotient $G / P$ is a rational complete homogeneous variety. Let $H$ be any complex algebraic group. A holomorphic principal $H$-bundle $E_{H} \longrightarrow G / P$ is called homogeneous if the left-translation action of $G$ on $G / P$ lifts to an action of $G$ on $E_{H}$ that commutes with the right action of $H$. The category of holomorphic homogeneous principal $H$-bundles coincides with the category of algebraic homogeneous principal $H$-bundles (see Lemma 2.1).

Now assume that $H$ is reductive. Fix a finite dimensional faithful $H$-module $V$. The following is the main result proved here (see Theorem 2.2).

Theorem 1.1. A principal $H$-bundle $E_{H} \longrightarrow G / P$ is homogeneous if and only if the associated vector bundle $E_{H} \times{ }^{H} V$ is homogeneous.

The method of proof of Theorem 1.1 yields the following (see Lemma 4.1):

Lemma 1.2. A principal $H$-bundle $E_{H} \longrightarrow G / P$ is homogeneous if and only if its adjoint vector bundle $\operatorname{ad}\left(E_{H}\right)$ is homogeneous.

Application 1.3. In Sa2], Sato proved that any infinitely extendable vector bundle on a nested sequence of homogeneous spaces is homogeneous (see [Sa2, p. 171, Main Theorem I] and [Sa2, p. 171, Main Theorem II] for the details). In view of Theorem 2.2, we conclude that the results of [Sa2] extend to principal bundles. In particular, any principal $H$-bundle on an infinite Grassmannian is homogeneous (see also [Sa1] and [DP]). Similarly, the results of Penkov and Tikhomirov (see [PT1], [PT2]) extend to principal bundles.

2000 Mathematics Subject Classification. 14L30, 14F05, $14 \mathrm{M} 17$.

Key words and phrases. Homogeneous bundle, principal bundle, homogeneous space. 


\section{Homogeneous principal Bundles And homogeneous Vector BundLES}

Let $G$ be a semisimple and simply connected linear algebraic group defined over $\mathbb{C}$, and let $P \subset G$ be a proper parabolic subgroup. So

$$
M:=G / P
$$

is a rational complete homogeneous variety. In the following, all morphisms, as well as all bundles on $M$, are supposed to be in the holomorphic category; they eventually may also be algebraic.

The left translation action of $G$ on itself defines an action of $G$ on the quotient space $M$. For any $g \in G$, let

$$
f_{g}: M \longrightarrow M
$$

be the holomorphic automorphism given by the action of $g$.

Let $H$ be a linear algebraic group defined over $\mathbb{C}$. A principal $H$-bundle $E_{H}$ on $M$ is called homogeneous if the action of $G$ on $M$ lifts to a holomorphic action of $G$ on $E_{H}$ that commutes with the right action of $H$. Equivalently, $E_{H}$ is the extension of structure group of the principal $P$-bundle $G \longrightarrow G / P$ by a homomorphism $P \longrightarrow H$.

A vector bundle $F$ on $M$ is called homogeneous if the action of $G$ on $M$ lifts to an action of $G$ on the total space of $F$ which is linear on the fibers. Thus a vector bundle of rank $n$ over $M$ is homogeneous if and only if the corresponding principal GL $(n, \mathbb{C})$-bundle is homogeneous.

The following lemma shows that homogeneous principal $H$-bundles are algebraically homogeneous.

Lemma 2.1. Let $E_{H} \longrightarrow M$ be a homogeneous principal $H$-bundle. Then the action of $G$ on $M$ lifts to an algebraic action of $G$ on $E_{H}$ satisfying the condition that it commutes with the right action of $H$.

Proof. Since $E_{H}$ is homogeneous, for each $g \in G$, the pulled back principal $H$-bundle $f_{g}^{*} E_{H}$ is holomorphically isomorphic to $E_{H}$, where $f_{g}$ is constructed in (2.2). Therefore, $f_{g}^{*} E_{H}$ is algebraically isomorphic to $E_{H}$ for all $g \in G$. Now from Proposition 3.1 of [Bi] we conclude that the action of $G$ on $M$ lifts to an algebraic action of $G$ on $E_{H}$ satisfying the condition that it commutes with the right action of $H$.

Let $H$ be a connected reductive linear algebraic group defined over $\mathbb{C}$. Fix a finite dimensional complex representation

$$
\rho_{0}: H \longrightarrow \mathrm{GL}(V)
$$

such that $\operatorname{kernel}\left(\rho_{0}\right)$ is a finite group. So the homomorphism of Lie algebras induced by $\rho$

$$
\mathfrak{h}:=\operatorname{Lie}(H) \longrightarrow \operatorname{End}(V)
$$

is injective.

Let $E_{H} \longrightarrow M$ be a principal $H$-bundle. Let

$$
E_{V}:=E_{H} \times{ }^{H} V \longrightarrow M
$$


be the vector bundle associated to $E_{H}$ for the $H$-module $V$ in (2.3).

Theorem 2.2. Assume that the associated vector bundle $E_{V}$ is homogeneous. Then the principal $H$-bundle $E_{H}$ is homogeneous.

\section{Proof of Theorem 2.2}

Let $E_{\mathrm{GL}(V)} \longrightarrow M$ be the principal $\mathrm{GL}(V)$-bundle corresponding to $E_{V}$. By assumption, we have an action $G \times E_{\mathrm{GL}(V)} \longrightarrow E_{\mathrm{GL}(V)}$ of $G$ on $E_{\mathrm{GL}(V)}$ that commutes with the action of $\mathrm{GL}(V)$ making $E_{\mathrm{GL}(V)}$ a homogeneous principal $\mathrm{GL}(V)$-bundle. Our aim is to prove that $E_{H}$ is homogeneous.

Let $\operatorname{At}\left(E_{\mathrm{GL}(V)}\right)$ (respectively, $\operatorname{At}\left(E_{H}\right)$ ) be the Atiyah bundle over $M$ for the principal $\mathrm{GL}(V)$-bundle $E_{\mathrm{GL}(V)}$ (respectively, principal $H$-bundle $\left.E_{H}\right)$. We recall that $\operatorname{At}\left(E_{\mathrm{GL}(V)}\right)$ (respectively, $\operatorname{At}\left(E_{H}\right)$ ) is the holomorphic vector bundle defined by the sheaf of $\mathrm{GL}(V)$ invariant vector fields on $E_{\mathrm{GL}(V)}$ (respectively, $H$-invariant vector fields on $E_{H}$ ); see [At]. Using the properties of the Atiyah bundle and the injectivity of the homomorphism in (2.3) we have the following diagram with exact rows

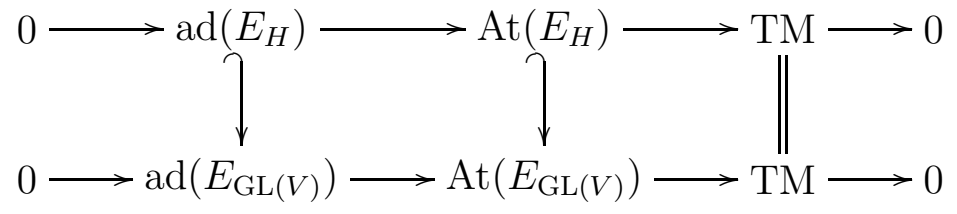

Since $H$ is reductive, the homomorphism of $H$-modules in (2.3) splits. In other words, there is a submodule $S$ of the $H$-module $\operatorname{End}(V)$ such that the natural homomorphism

$$
\mathfrak{h} \oplus S \longrightarrow \operatorname{End}(V)
$$

is an isomorphism of $H$-modules. Fix such a direct summand $S$, and let

$$
E_{S}:=E_{H} \times{ }^{H} S
$$

be the vector bundle over $M$ associated to the principal $H$-bundle $E_{H}$ for the $H$-module $S$. From (3.2) we obtain an isomorphism

$$
\operatorname{ad}\left(E_{H}\right) \oplus E_{S} \cong \operatorname{ad}\left(E_{\mathrm{GL}(V)}\right)
$$

of associated vector bundles. Hence from (3.1) we have the commutative diagram

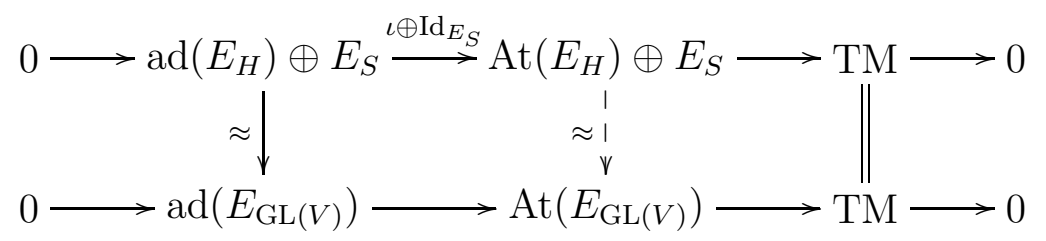

where $\iota: \operatorname{ad}\left(E_{H}\right) \longrightarrow \operatorname{At}\left(E_{H}\right)$ is the inclusion in (3.1). Let

$$
\sigma: H^{0}\left(M, \operatorname{At}\left(E_{\mathrm{GL}(V)}\right)\right) \longrightarrow H^{0}\left(M, \operatorname{At}\left(E_{H}\right)\right)
$$

be the surjective homomorphism induced by the projection $\operatorname{At}\left(E_{\mathrm{GL}(V)}\right) \longrightarrow \operatorname{At}\left(E_{H}\right)$ constructed from (3.3). 
Let $\mathfrak{g}$ denote the Lie algebra of $G$. Let

$$
\varphi: \mathfrak{g} \longrightarrow H^{0}\left(M, \operatorname{At}\left(E_{\mathrm{GL}(V)}\right)\right) \text { and } \varphi_{H}: \mathfrak{g} \longrightarrow H^{0}\left(M, \operatorname{At}\left(E_{H}\right)\right)
$$

be the homomorphisms of Lie algebras given by the actions of $G$ on $E_{\mathrm{GL}(V)}$ and $E_{H}$ respectively. Note that

$$
\varphi_{H}=\sigma \circ \varphi
$$

where $\sigma$ is constructed in (3.4). We have the following commutative diagram of Lie algebras

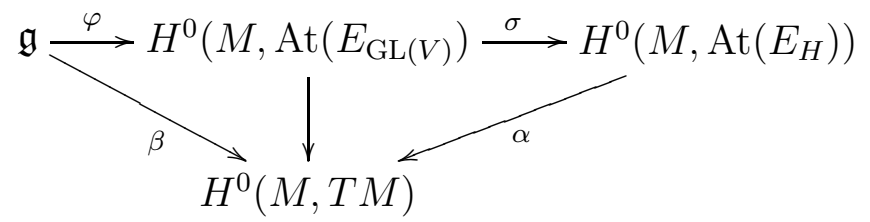

where $\beta$ is the injective Lie algebra homomorphism induced by the natural action of $G$ on $G / P$, and $\varphi, \sigma$ are defined above; the remaining two homomorphisms are obtained from (3.1). Consequently, $\beta(\mathfrak{g}) \subset \alpha\left(H^{0}\left(M, \operatorname{At}\left(E_{H}\right)\right)\right)$. Defining

$$
H^{0}\left(\widetilde{M, \operatorname{At}}\left(E_{H}\right)\right):=\alpha^{-1}(\beta(\mathfrak{g}))
$$

from (3.5) and (3.1) we have a short exact sequence of Lie algebras

$$
0 \longrightarrow H^{0}\left(M, \operatorname{ad}\left(E_{H}\right)\right) \longrightarrow H^{0}\left(\widehat{M, \operatorname{At}}\left(E_{H}\right)\right) \stackrel{\alpha}{\longrightarrow} \mathfrak{g} \longrightarrow 0
$$

Since $\mathfrak{g}$ is semisimple, there is a homomorphism of Lie algebras

$$
\widehat{\alpha}: \mathfrak{g} \longrightarrow H^{0}\left(\widehat{M, \operatorname{At}}\left(E_{H}\right)\right)
$$

such that $\alpha \circ \widehat{\alpha}=\operatorname{Id}_{\mathfrak{g}}$; see [Bo, p. 91, Corollaire 3]. Fix such a splitting $\widehat{\alpha}$.

Let $\mathcal{G}\left(E_{H}\right)$ denote the group of all biholomorphisms of $E_{H}$ that commute with the right action of $H$. It is a complex Lie group, and its Lie algebra coincides with $H^{0}\left(M, \operatorname{At}\left(E_{H}\right)\right)$ (see [Bi] for a proof). The group $G$ being simply connected, the homomorphism of Lie algebras $\widehat{\alpha}$ in (3.7) lifts to a homomorphism

$$
\rho: G \longrightarrow \mathcal{G}\left(E_{H}\right) \text {. }
$$

Since $\alpha \circ \widehat{\alpha}=\operatorname{Id}_{\mathfrak{g}}$, it follows that the action of $G$ on $E_{H}$ defined by $\rho$ makes $E_{H}$ a homogeneous principal $H$-bundle. This completes the proof of Theorem 2.2 .

\section{Adjoint Bundle CRITERION}

The above proof of Theorem 2.2 also gives the following lemma.

Lemma 4.1. Assume that the adjoint vector bundle $\operatorname{ad}\left(E_{H}\right)$ is homogeneous. Then $E_{H}$ is homogeneous.

Proof. Define $Z:=H /[H, H]$. Any holomorphic principal $Z$-bundle on $G / P$ is homogeneous because $Z$ is a product of copies of $\mathbb{C}^{*}$ and $G$ is simply connected (so any line bundle on $G / P$ is homogeneous). The $H$-module $\mathfrak{h}$ decomposes as

$$
\mathfrak{h}=[\mathfrak{h}, \mathfrak{h}] \oplus z(\mathfrak{h}),
$$


where $z(\mathfrak{h})$ is the Lie algebra of $Z$. The adjoint homomorphism $\mathfrak{h} \longrightarrow \operatorname{End}_{\mathbb{C}}(\mathfrak{h})$ is injective. So, the homomorphism of Lie algebras corresponding to the homomorphism

$$
H \longrightarrow \mathrm{GL}(\mathfrak{h}) \times Z=: \widetilde{H}
$$

is injective. Let $E_{\widetilde{H}}$ be the principal $\widetilde{H}$-bundle on $M$ obtained by extending the structure group of $E_{H}$ using the above homomorphism. Since $\operatorname{ad}\left(E_{H}\right)$ and all holomorphic line bundles on $G / P$ are homogeneous, and $Z$ is a product of copies of $\mathbb{C}^{*}$, it follows that $E_{\widetilde{H}}$ is homogeneous. After replacing the principal bundle $E_{\mathrm{GL}(V)}$ by $E_{\widetilde{H}}$, it is straight-forward to check that the proof of Theorem 2.2 also gives a proof of the lemma.

Remark 4.2. Let $E_{H}$ be a principal $H$-bundle on $M$, and let $H \longrightarrow \mathrm{GL}(V)$ be a faithful representation as in (2.2). If the associated vector bundle $E_{V}$ is trivial, then it can be shown that $E_{H}$ itself is trivial. To prove this, consider the induced morphism $E_{H} \longrightarrow E_{\mathrm{GL}(V)}$. The principal $\mathrm{GL}(V)$-bundle $E_{\mathrm{GL}(V)}$ is trivial because $E_{V}$ is trivial. Since $M$ is complete and $\mathrm{GL}(V) / H$ affine, there are no non-constant maps from $M$ to $\mathrm{GL}(V) / H$. This implies that there are trivializing sections of $E_{\mathrm{GL}(V)}$ which factor through $E_{H}$. By this remark, the result of [PT1] on the triviality of vector bundles on twisted indGrassmannians can be extended to principal bundles. (See [BCT] for principal bundles on projective spaces.)

\section{REFERENCES}

[At] M. F. Atiyah, Complex analytic connections in fibre bundles, Trans. Amer. Math. Soc. 85 (1957) 181-207.

[Bi] I. Biswas, Homogeneous principal bundles and stability, Forum Math. 22 (2010) 603-617.

[BCT] I. Biswas, I. Coandă and G. Trautmann, A Babylonian tower theorem for principal bundles over projective spaces, Jour. Math. Kyoto Univ. 49 (2009) 69-82.

[Bo] N. Bourbaki, Éléments de mathématique. XXVI. Groupes et algèbres de Lie. Chapitre 1: Algèbres de Lie, Actualités Sci. Ind. No. 1285, Hermann, Paris, 1960.

[DP] J. Donin and I. Penkov, Finite rank vector bundles on inductive limits of Grassmannians, Int. Math. Res. Not. (2003) 1871-1887.

[PT1] I. Penkov and A. S. Tikhomirov, Triviality of vector bundles on sufficiently twisted indGrassmannians, arXiv:0706.3912.

[PT2] I. Penkov and A. S. Tikhomirov, Rank 2 vector bundles on ind-Grassmannians, arXiv:0710.0905.

[Sa1] E.-i. Sato, On the decomposability of infinitely extendable vector bundles on projective spaces and Grassmann varieties, Jour. Math. Kyoto Univ. 17 (1977) 127-150.

[Sa2] E.-i. Sato, On infinitely extendable vector bundles on $G / P$, Jour. Math. Kyoto Univ. 19 (1979) $171-189$.

School of Mathematics, Tata Institute of Fundamental Research, Homi Bhabha Road, BOMBAY 400005, INDIA

E-mail address: indranil@math.tifr.res.in

FB Mathematik, Universität Kaiserslautern, Postfach 3049, D-67653 Kaiserslautern, GERMANY

E-mail address: trm@mathematik.uni-kl.de 